\title{
Comparison of Clinical Outcomes of Different Rates of Infusion in Caudal Epidural Steroid Injection: A Randomized Controlled Trial
}

\author{
Satit Thiengwittayaporn, Punsang Koompong, Supat Khamrailert, Pumibal Wetpiriyakul \\ Department of Orthopaedics, Faculty of Medicine Vajira Hospital, Navamindradhiraj University, Bangkok, Thailand
}

\begin{abstract}
Study Design: Prospective randomized controlled equivalence trial.
Purpose: To compare the clinical outcomes between patients with lumbosacral radiculopathy that received caudal epidural steroid injection (CESI) at injection rates of $40 \mathrm{~mL} / \mathrm{min}$ (fast rate) and $20 \mathrm{~mL} / \mathrm{min}$ (slow rate) and to identify the complications associated with these different CESI rates.

Overview of Literature: CESI is widely used for chronic low back pain with lumbar radiculopathy. However, the influence of CESI rates on clinical outcomes has not been well studied.

Methods: Ninety patients were randomized into two CESI groups. Two patients did not complete the study. Eighty-eight were included in the final analysis: 44 patients were in the fast infusion group, and 44 patients were in the slow infusion group. Intragroup and intergroup comparisons were conducted with regard to the Visual Analog Scale (VAS), Roland 5-point pain scale, standing tolerance test, walking tolerance test, and patient satisfaction scale at pre-injection, 2 weeks, 6 weeks, and 12 weeks post-injection. Complications associated with the different rates were recorded.

Results: Both groups demonstrated clinical improvement in all parameters, except for VAS, after injection. There were no statistically significant differences in any outcomes at each time point between the two groups. One patient in the fast rate group and no patients in the slow rate group experienced nausea and vomiting after injection $(p=0.320)$. Eight patents in the fast rate group and two patients in the slow rate group experienced pain at the injection site $(p=0.044)$.

Conclusions: Although there were no significant differences between injection rates in the short-term clinical improvement outcomes, the fast injection rate group experienced more pain at the injection site, suggesting that the use of the slow injection rate may be considered.
\end{abstract}

Keywords: Caudal epidural steroid injection; Epidural injections; Lumbosacral radiculopathy; Radiculopathy

\section{Introduction}

Caudal epidural steroid injection (CESI) is a widely used interventional treatment for chronic low back pain in the presence or absence of lumbar radiculopathy. It is an effective and relatively safe procedure when performed with appropriate care under fluoroscopic visualization $[1,2]$. To improve the efficacy of these injections, many studies have

Received Dec 10, 2019; Revised Feb 15, 2020; Accepted Mar 18, 2020

Corresponding author: Satit Thiengwittayaporn

Department of Orthopaedics, Faculty of Medicine Vajira Hospital, Navamindradhiraj University, 681 Samsen Rd, Dusit, Bangkok,10300 Thailand

Tel: +66-22443376, Fax: +66-22436106, E-mail: vajira@nmu.ac.th 
focused on potential strategies to enhance the cephalic migration of drug delivery. Theoretically, the spread of solutions injected into the epidural space depends on the volume and rate of infusion at the injection site [3].

The effect of epidural injections on intracranial pressure (ICP) has been studied, and it is known that epidural injections, at least transiently, increase ICP. The literature reveals that spinal cord compression following rapid injections into the epidural space may cause large increases in intraspinal pressure with a risk of cerebral hemorrhage, visual disturbances, headache, and compromise of spinal cord blood flow [4]. Consequently, a slow injection rate that is clinically effective should be used. However, the volume of solution used and the rate of injection vary widely in different centers. Studies comparing the effect of different rates of injection are scarce and inconclusive.

Therefore, in this study, we conducted a randomized controlled trial to (1) compare the clinical outcomes between patients with lumbosacral radiculopathy that received CESI at injection rates of $40 \mathrm{~mL} / \mathrm{min}$ (fast rate) and $20 \mathrm{~mL} / \mathrm{min}$ (slow rate), and (2) identify the complications associated with these different CESI rates. We hypothesized that the slower infusion rate would demonstrate equivalent short-term clinical outcomes compared to the faster infusion rate.

\section{Materials and Methods}

\section{Study design}

The study was a prospective, randomized controlled equivalence trial. The study was conducted in accordance with the principles of the Declaration of Helsinki. Approval for the study was obtained from Navamindradhiraj University Review Board (COA 74/2559). This study was registered in clinicaltrial.gov and all participants provided informed consent prior to the study.

\section{Participants}

1) Inclusion criteria

All patients who were evaluated at our outpatient clinic who were above 20 years old with chronic low back pain and radicular symptoms attributable to lumbar disk disease and/or spinal stenosis on a prior magnetic resonance scan were considered for inclusion.

\section{2) Exclusion criteria}

Patients were excluded from the trial if they had a previous lumbar surgery, previous CESI, coagulopathy, or a skin infection at the injection site.

\section{Recruitment}

All patients were initially screened with regard to the selection criteria before randomization. If the inclusion criteria were met and the informed consent form was signed, the patient was scheduled for randomization.

\section{Sample size}

The trial was conducted as an equivalence study. Sample size was calculated based on reduction of Visual Analog Scale (VAS). A minimal clinical difference change of 0.5 (d) was set from an expert's opinion. Data from a previous study indicated that patients typically reported a change of 2.45 points (standard deviation $[S D]=0.91$ ) on the VAS at 2 weeks post-injection [5]. With a SD $(\sigma)$ of the VAS of $0.91, \delta=\mathrm{d} / \sigma, \delta=0.80$, to achieve an alpha of 0.05 and beta of 0.20 with $80 \%$ power [6], 32 patients would be required for each group of the trial. Taking into account a $20 \%$ potential loss to follow-up, at least 40 patients per group would be considered for recruitment.

\section{Randomization}

Site-specific randomization lists were computer-generated using randomly permuted blocks of four and were concealed from the researchers by a senior data manager who was not involved in the study. This trial used a prospective, randomized, and outcome-blinded design, in which all outcome assessments were made by a research assistant blinded to the treatment allocation and patient information. Forty-five participants selected from the 90 patients included were randomly assigned to each group (Fig. 1).

\section{Allocation concealment}

Patient randomization was performed by another coordinator, without the knowledge of the physicians and participants. Study participants were mixed with routine treatment participants. A statistician that was not involved with patient care assembled the data. 


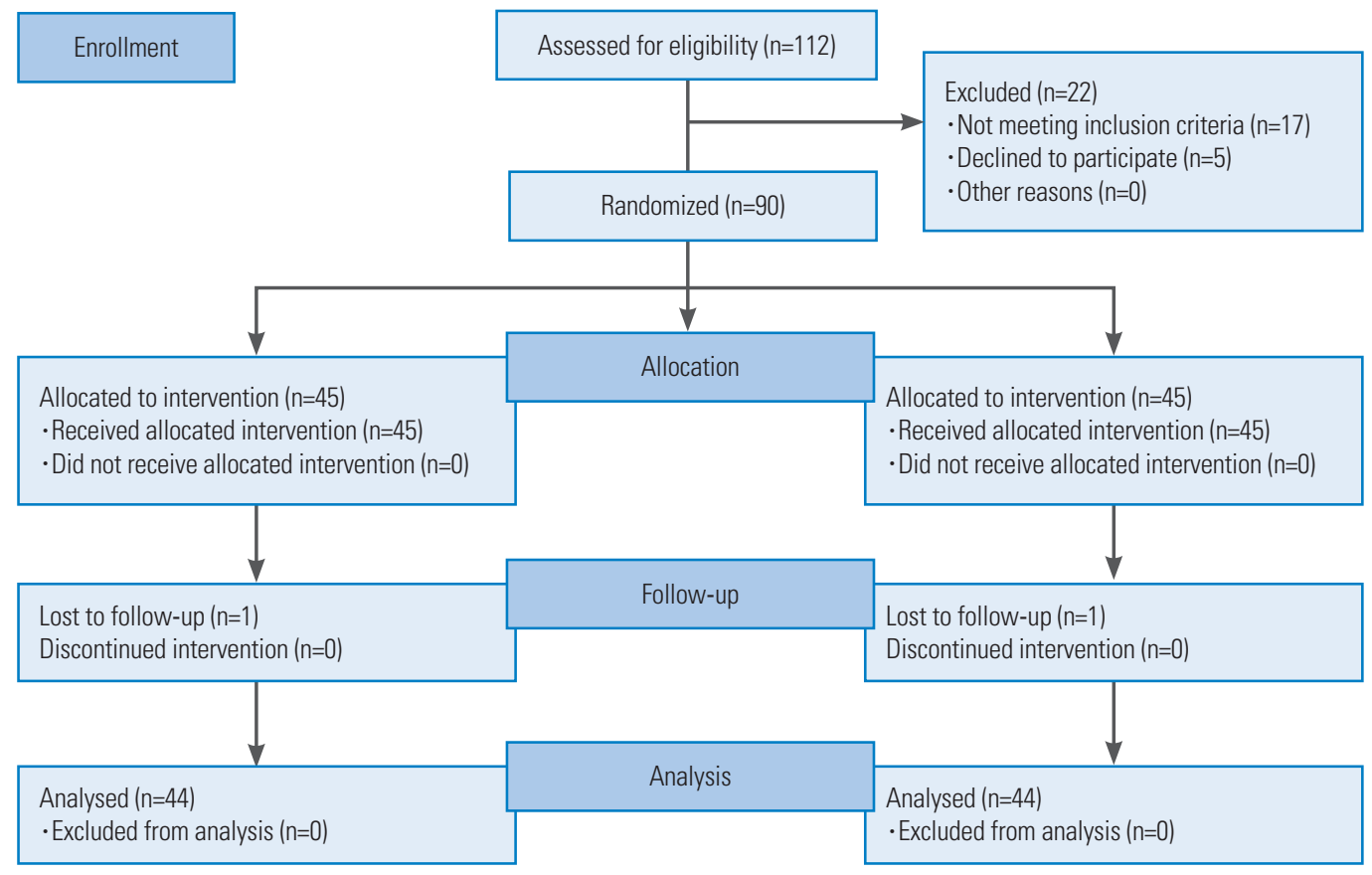

Fig. 1. Flowchart of patients through the trial involving $40 \mathrm{~mL} / \mathrm{min}$ (fast rate group) or $20 \mathrm{~mL} / \mathrm{min}$ (slow rate group) for caudal epidural steroid injection.

\section{Intervention}

Eligible patients were randomized into two groups as follows: a fast infusion rate group $(40 \mathrm{~mL} / \mathrm{min}, 45$ patients) and a slow infusion rate group $(20 \mathrm{~mL} / \mathrm{min}, 45$ patients). Clinical variables including age, sex, body weight, height, body mass index, level of spine involvement, grading of spinal stenosis on magnetic resonance imaging [5,6], and duration of symptoms were recorded prior to the intervention. Clinical parameters including VAS [7], Roland 5-point pain scale, standing tolerance test, walking tolerance test, and patient satisfaction scale were also evaluated before the intervention [8] (Table 1).

All injections were administered by the same surgeon, a spine specialist, under fluoroscopic control to ensure accurate needle placement. All patients were positioned prone during the procedure. A standardized technique was used in all procedures. A wedge-shaped pillow was placed under the hips to tilt the pelvis and bring the sacral hiatus into greater prominence. The sacrococcygeal area was prepared using an iodine-based antiseptic solution (povidone-iodine USP solution). The surgeon then used a sterile gloved finger of the dominant hand to localize the tip of the coccyx through palpation and applied an anesthetic of $2 \%$ lidocaine. An 18G, 90-mm spinal needle
Table 1. Measurement of outcomes

\begin{tabular}{|c|c|}
\hline Variable & Score \\
\hline Visual Analog Scale & $0-10$ \\
\hline Roland 5-point pain scale & $\begin{array}{l}\text { 0: Absence of pain } \\
\text { 1: Little pain } \\
\text { 2: Moderate pain } \\
\text { 3: Bad pain } \\
\text { 4: Very bad pain } \\
\text { 5: Almost unbearable pain }\end{array}$ \\
\hline Standing tolerance test (min) & $\begin{array}{l}0: 0-5 \\
1: 5-10 \\
2: 10-30 \\
3: 30-60 \\
4:>60\end{array}$ \\
\hline Walking tolerance test feet (m) & $\begin{array}{l}0: 0-15.24 \\
1: 15.5448-60.96 \\
2: 61.2648-152.4 \\
3: 152.7048-804.672 \\
4:>804.672\end{array}$ \\
\hline Patient satisfaction scale & $\begin{array}{l}\text { 4: Completely better } \\
\text { 3: Somewhat better } \\
\text { 2: Same } \\
\text { 1: Slightly worse } \\
\text { 0: Worse }\end{array}$ \\
\hline
\end{tabular}

(Quincke type point; B. Braun Melsungen AG, Melsungen, Germany) was guided via fluoroscopy (Philips BV libra c-arm, Eindhoven, the Netherlands) to the midline of the sacral hiatus. A lateral fluoroscopic view was used to 
confirm that the needle was in the caudal epidural space. Aspirations were routinely performed. The same injectate was used in all patients and consisted of $18 \mathrm{~mL}$ of normal saline and $2 \mathrm{~mL}$ of triamcinolone acetonide $(40 \mathrm{mg} /$ $\mathrm{mL}$ ). The injection rate was controlled by a $50-\mathrm{mL}$ syringe pump (Syringe pump; Treat Med Co., Bangkok, Thailand) that provided $20 \mathrm{~mL}$ in 30 seconds for the fast rate group $(40 \mathrm{~mL} / \mathrm{min})$ and $20 \mathrm{~mL}$ in 60 seconds for the slow rate group $(20 \mathrm{~mL} / \mathrm{min})$.

All patients were monitored by pulse oximetry and blood pressure after the procedure and were observed for any complications by a physician, who did not perform the injection, and by a registered nurse for 30 minutes before discharge.

\section{Patient follow-up for outcomes evaluation}

Patients were evaluated at 2, 6, and 12 weeks post-injection. Clinical outcomes were evaluated using the VAS, Roland 5-point pain scale, standing tolerance test, walking tolerance test, and patient satisfaction scale [8]. VAS has a horizontal, 100-mm-long line, with "no pain" recorded on the left side (score: 0 ) and "pain as bad as it could be" on the right side (score: 10). Patients were asked to place a mark on the line that corresponded to their current level of pain. The VAS score was then determined by measuring the millimeters between the left endpoint and the point that the patient marked.

\section{Quality control}

Before the trial, all staff involved was trained regarding patient inclusion and exclusion criteria, data collection, and intervention methods. During the trial, supervisors checked the case report forms and intervention processes. Dropouts, withdrawals, reasons for withdrawal, and the compliance of all patients were recorded in detail throughout the treatment and follow-up period.

\section{Statistical analysis}

The demographic data and clinical outcomes of both groups were compared using independent samples $t$-tests and chi-square tests. Shapiro-Wilk tests were used to assess normally distributed variables. Unpaired Student $t$-tests were used to compare the groups, whereas a paired Student $t$-tests were used for multiple time point com- parisons. A $p$-value of $<0.05$ was considered to indicate a statistically significant difference. IBM SPSS ver. 23.0 (IBM Corp., Armonk, NY, USA) was used for all analyses.

\section{Results}

In this randomized controlled trial, 45 patients were recruited in each group, with both groups recording a loss to follow-up of one patient each. One patient from the fast rate group was lost to follow-up because of an ankle fracture. The patient from the slow rate group was lost to follow-up due to relocation. The patients' demographic data and pre-injection clinical parameters revealed no significant differences between the groups (Table 2).

To determine if a statistically significant difference existed before and after treatment, a paired sample $t$-test was performed to evaluate the difference between pre- and post-injection values of the outcome parameters. Irrespective of the measured outcome, there was a statistically significant improvement comparing the pre-injection values and the initial and 2, 6, and 12-week follow-up visits in all clinical outcomes except for VAS (Tables 3, 4).

When clinical outcomes were compared between the two groups at each time point, there were no significant differences in all outcomes. This included VAS, Roland 5-point pain scale, standing tolerance test, walking tolerance test, and patient satisfaction scale (Table 5).

One patient in the fast rate group and no patients in the slow rate group experienced nausea and vomiting after injection $(p=0.320)$. Eight patents in the fast rate group and two patients in the slow rate group experienced pain at the infiltration site $(p=0.044)$ (Table 6).

\section{Discussion}

CESI is a well-accepted technique for the treatment of radicular low back pain. However, it is essential to underline the subtle but important distinction between the physical spread of a solution in the epidural space and its clinical efficacy. The former can be measured using radiological evidence of the distribution of solutions in the epidural space, but the latter is recognized by the clinical outcomes.

The spread of solutions injected into the epidural space depends on the injection volume and rate at the injection site [3]. Regarding the injection volume, it is not linearly volume dependent despite the increasing volume of the injectate. The leakage of solution and "staring effect" of 
Table 2. Demographics and baseline characteristics

\begin{tabular}{|c|c|c|c|}
\hline Variable & Fast rate $(\mathrm{N}=44)$ & Slow rate $(\mathrm{N}=44)$ & $p$-value \\
\hline Age (yr) & $64.3 \pm 10.2$ & $64.9 \pm 9.2$ & $0.769^{\mathrm{a})}$ \\
\hline Gender & & & $0.565^{\mathrm{bl}}$ \\
\hline Female & $38(86.4)$ & $36(81.8)$ & \\
\hline Male & $6(13.6)$ & $8(18.2)$ & \\
\hline Body weight (kg) & $63.4 \pm 11.3$ & $64.8 \pm 14.7$ & $0.601^{a)}$ \\
\hline Height $(\mathrm{cm})$ & $155.6 \pm 6.9$ & $154.0 \pm 15.7$ & $0.525^{\text {a) }}$ \\
\hline Body mass index $\left(\mathrm{kg} / \mathrm{m}^{2}\right)$ & $26.2 \pm 4.3$ & $31.0 \pm 19.9$ & $0.118^{\mathrm{al}}$ \\
\hline Level of involvement & $1.9(1-4)$ & $1.8(1-4)$ & $0.365^{\text {a) }}$ \\
\hline \multicolumn{4}{|c|}{ Grading of spinal stenosis on magnetic resonance imaging $(\%)$} \\
\hline L1-2 (grade 0/1/2/3) & 20.1/39.9/20.0/20.0 & $42.9 / 42.9 / 14.3 / 0.0$ & $0.589^{\mathrm{a})}$ \\
\hline L2-3 (grade 0/1/2/3) & 20.0/40.0/19.0/21.0 & $14.3 / 57.1 / 28.6 / 0.0$ & $0.634^{a)}$ \\
\hline L3-4 (grade 0/1/2/3) & $0.0 / 59.0 / 0.0 / 41.0$ & $0.0 / 57.1 / 14.3 / 28.6$ & $0.659^{a)}$ \\
\hline L4-5 (grade 0/1/2/3) & $0.0 / 0.0 / 17.7 / 82.3$ & $0.0 / 42.9 / 0.0 / 57.1$ & $0.152^{\mathrm{al}}$ \\
\hline L5-S1 (grade 0/1/2/3) & 21.1/39.9/0.0/39.0 & $14.3 / 42.9 / 14.3 / 28.3$ & $0.827^{\text {a) }}$ \\
\hline Duration of symptom (mo) & $17.2 \pm 25.3$ & $20.0 \pm 22.4$ & $0.580^{\mathrm{a})}$ \\
\hline Visual Analog Scale & $7.4 \pm 1.9$ & $7.7 \pm 1.8$ & $0.421^{\text {a) }}$ \\
\hline Roland 5-point pain scale & $3.6 \pm 1.1$ & $3.7 \pm 1.1$ & $0.623^{\mathrm{a})}$ \\
\hline Standing tolerance & $0.5 \pm 0.8$ & $0.4 \pm 0.7$ & $0.591^{\text {a) }}$ \\
\hline Walking tolerance & $1.1 \pm 1.1$ & $1.0 \pm 1.1$ & $0.780^{\mathrm{a})}$ \\
\hline Patients satisfaction & $0.3 \pm 0.5$ & $0.4 \pm 0.5$ & $0.514^{\mathrm{a})}$ \\
\hline
\end{tabular}

Values are presented as mean \pm standard deviation, number $(\%)$, or median (range).

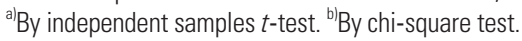

Table 3. Outcomes of fast rate group at pre-injection, 2 weeks, 6 weeks, and 12 weeks after the injection

\begin{tabular}{lccccc} 
Outcome & Pre-injection & 2 wk & 6 wk & \multicolumn{1}{c}{12 wk } & $p$-value ${ }^{\text {al }}$ \\
Visual Analog Scale & $7.4 \pm 1.9$ & $3.8 \pm 2.6$ & $3.9 \pm 2.8$ & $4.6 \pm 3.1$ & 0.054 \\
\hline Roland 5-point pain scale & $3.6 \pm 1.1$ & $1.5 \pm 1.2$ & $1.6 \pm 1.2$ & $1.9 \pm 1.4$ & $<0.001^{*}$ \\
\hline Standing tolerance & $0.5 \pm 0.8$ & $2.1 \pm 1.6$ & $2.1 \pm 1.6$ & $1.8 \pm 1.6)$ & $<0.001^{*}$ \\
Walking tolerance & $1.1 \pm 1.1$ & $2.9 \pm 1.3$ & $3.0 \pm 1.3$ & $2.7 \pm 1.4$ & $<0.001^{*}$ \\
\hline Patients satisfaction & $0.3 \pm 0.5$ & $2.9 \pm 0.8$ & $2.7 \pm 1.1$ & $2.4 \pm 1.3$ & $<0.001^{*}$ \\
\hline
\end{tabular}

Values are presented as mean \pm standard deviation.

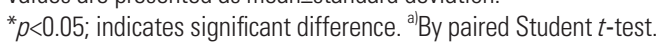

Table 4. Outcomes of slow rate group at pre-injection, 2 weeks, 6 weeks, and 12 weeks after the injection

\begin{tabular}{lccccc} 
Outcome & Pre-injection & 2 wk & 6 wk & \multicolumn{1}{c}{12 wk } & p-value \\
Visual Analog Scale & $7.7 \pm 1.8$ & $3.6 \pm 2.6$ & $4.7 \pm 3.3$ & $5.1 \pm 3.2$ & 0.156 \\
Roland 5-point pain scale & $3.7 \pm 1.1$ & $1.1 \pm 0.9$ & $2.1 \pm 1.6$ & $2.1 \pm 1.4$ & $<0.001^{*}$ \\
Standing tolerance & $0.4 \pm 0.7$ & $1.5 \pm 1.5$ & $1.6 \pm 1.7$ & $1.4 \pm 1.5$ & $0.001^{*}$ \\
Walking tolerance & $1.0 \pm 1.1$ & $2.8 \pm 1.3$ & $2.4 \pm 1.6$ & $2.5 \pm 1.5$ & $<0.001^{*}$ \\
\hline Patients satisfaction & $0.4 \pm 0.5$ & $3.2 \pm 0.8$ & $2.4 \pm 1.3$ & $2.3 \pm 1.3$ & $<0.001^{*}$ \\
\hline
\end{tabular}

Values are presented as mean \pm standard deviation.

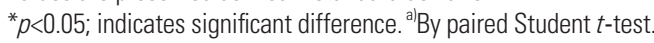


Table 5. Comparison of five outcome measurements between the two groups

\begin{tabular}{|c|c|c|c|}
\hline Outcome & Fast rate $(\mathrm{N}=44)$ & Slow rate $(\mathrm{N}=44)$ & $p$-value ${ }^{\text {a) }}$ \\
\hline \multicolumn{4}{|c|}{ Visual Analog Scale } \\
\hline Pre-injection & $7.4 \pm 1.9$ & $7.7 \pm 1.8$ & 0.421 \\
\hline $2 w k$ & $3.8 \pm 2.6$ & $3.6 \pm 2.6$ & 0.770 \\
\hline 6 wk & $3.9 \pm 2.8$ & $4.7 \pm 3.3$ & 0.240 \\
\hline 12 wk & $4.6 \pm 3.1$ & $5.1 \pm 3.2$ & 0.490 \\
\hline \multicolumn{4}{|c|}{ Roland 5-point pain scale } \\
\hline Pre-injection & $3.6 \pm 1.1$ & $3.7 \pm 1.1$ & 0.623 \\
\hline $2 w k$ & $1.5 \pm 1.2$ & $1.4 \pm 0.9$ & 0.093 \\
\hline 6 wk & $1.6 \pm 1.2$ & $2.1 \pm 1.6$ & 0.073 \\
\hline 12 wk & $1.9 \pm 1.4$ & $2.1 \pm 1.4$ & 0.595 \\
\hline \multicolumn{4}{|c|}{ Standing tolerance } \\
\hline Pre-injection & $0.5 \pm 0.8$ & $0.4 \pm 0.7$ & 0.591 \\
\hline $2 w k$ & $2.1 \pm 1.6$ & $1.5 \pm 1.5$ & 0.057 \\
\hline $6 w k$ & $2.1 \pm 1.6$ & $1.6 \pm 1.7$ & 0.152 \\
\hline 12 wk & $1.8 \pm 1.6$ & $1.4 \pm 1.5$ & 0.252 \\
\hline \multicolumn{4}{|c|}{ Walking tolerance } \\
\hline Pre-injection & $1.1 \pm 1.1$ & $1.0 \pm 1.1$ & 0.780 \\
\hline 2 wk & $2.9 \pm 1.3$ & $2.8 \pm 1.3$ & 0.747 \\
\hline 6 wk & $3.0 \pm 1.3$ & $2.4 \pm 1.6$ & 0.061 \\
\hline 12 wk & $2.7 \pm 1.4$ & $2.5 \pm 1.5$ & 0.720 \\
\hline \multicolumn{4}{|c|}{ Patients satisfaction } \\
\hline Pre-injection & $0.3 \pm 0.5$ & $0.4 \pm 0.5$ & 0.514 \\
\hline $2 w k$ & $2.9 \pm 0.8$ & $3.2 \pm 0.8$ & 0.090 \\
\hline 6 wk & $2.7 \pm 1.1$ & $2.4 \pm 1.3$ & 0.297 \\
\hline 12 wk & $2.4 \pm 1.3$ & $2.3 \pm 1.3$ & 0.674 \\
\hline
\end{tabular}

Values are presented as mean \pm standard deviation.

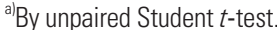

Table 6. Comparison of complications between groups

\begin{tabular}{lccc} 
Outcome & Fast rate $(\mathrm{N}=44)$ & Slow rate $(\mathrm{N}=44)$ & $p$-value \\
\hline Pain at injection site & 8 & 2 & $0.044^{*}$ \\
Nausea and vomiting & 1 & 0 & 0.320 \\
\hline
\end{tabular}

Values are presented as number of patients.

${ }^{*} p<0.05$; indicates significant difference.

the epidural space cause variations in the medication level achieved with the same volume [9]. To date, no data has shown that a specific volume is more efficacious than another, although a volumetric caudal epidural approach with as much as $50 \mathrm{~mL}$ has historically been advocated [10]. Kim et al. [11] injected $50 \mathrm{~mL}$ in sequential boluses of $10 \mathrm{~mL}$ under fluoroscopy and found that the first $10 \mathrm{~mL}$ of injection could reach as far as the mid to lower lumbar area consistently with a minimal incremental spread of injectate with increasing volumes. This was attributed to the "repainting" of the epidural space rather than a "building block" effect due to the mechanical and anatomical characteristics of the epidural space. In contrast, Murphy et al. [12] performed a magnetic resonance epidurography to assess the distribution of injectate using two different volumes during CESI. They found that a volume of 10 $\mathrm{mL}$ was unlikely to be effective for L5/S1 disease in nearly half of the patients and that at least $20 \mathrm{~mL}$ of injectate was necessary for any medication to reach the desired level, including L4/5 and above.

In terms of injection rate, few studies have investigated the effect of injection rate on the spread of solutions injected into the epidural space. Although Nishimura et al. [13] reported that the high pressure resulting from rapid injections failed to result in greater spread of injected solution and concluded that "speed of injection does not affect the spread of the solution," the study used only anesthetic solution injected at the lumbar region in the lateral decubitus position. Theoretically, rapid injection, like the use of large volumes, causes discomfort to the conscious patient and may result in loss of consciousness due to a pressure wave being transmitted through the cerebrospinal fluid. However, the only complication reported following epidural injection has been vision loss [14]. Retinal hemorrhages have been predominantly attributed to rapid epidural injections of high volumes, causing a sudden increase in ICP and increased retinal venous pressure [15]. To our knowledge, the effect of injection rate on the outcome of CESI has not been investigated.

Our study revealed a significant benefit for patients with lumbosacral radicular pain at 2, 6, and 12 weeks following fluoroscopic CESI regardless of injection rates. Except for VAS, the other outcome measures, including Roland 5-point pain scale, standing tolerance test, walking tolerance test, and patient satisfaction score, were significantly improved when compared with pre-injection values in both groups. Furthermore, there was no significant difference between the fast and slow injection groups in terms of outcomes measurement, with the most notable improvement occurring at 2 weeks post-injection in most categories of outcomes.

Although this study did not find significant differences in clinical improvement between the two injection rate groups after injection, the complications from pain and discomfort at the injection site were found more often in 
the fast rate infusion. Moreover, one patient in the fast rate group experienced nausea and vomiting that may be caused by a transient increase in ICP.

A fast rate of CESI is often used because it is believed to improve the cephalad spread of drug delivery and lead to better clinical outcomes. Our study showed no advantages regarding improvement of clinical outcomes after CESI with the faster rate of infusion compared to the slower rate. Our outcomes support the use of a slower infusion rate to minimize the risk of a transiently increased ICP.

The study has several limitations. Firstly, the most suitable speed of CESI injection was unclear. However, we used $40 \mathrm{~mL} / \mathrm{min}$ as a practical rate compared with the slower rate of $20 \mathrm{~mL} / \mathrm{min}$. Second, we limited our study period to the first 12 weeks after injection. We believe that any beneficial effects would be most notable within the immediate short term, and therefore, a period of 12 weeks seemed sufficient to test our hypothesis. Previous studies have shown that any benefit from a caudal epidural injection would occur in the immediate short-term period [16].

\section{Conclusions}

Our study found a significant improvement in short-term clinical discomfort for patients that underwent CESI under fluoroscopic guidance. Comparison between the clinical outcomes of faster $(40 \mathrm{~mL} / \mathrm{min})$ and slower $(20 \mathrm{~mL} /$ $\mathrm{min}$ ) injection rates revealed no significant differences in the short-term period. However, the faster injection rate group experienced more pain at the injection site, suggesting that the use of the slow injection rate might be considered.

\section{Conflict of Interest}

No potential conflict of interest relevant to this article was reported.

\section{Acknowledgments}

This project was supported by the Navamindradhiraj University Research Fund grant numbers CEU/C 043/2559. We would like to thank Ms Thikampa Wichai, for their help in collecting and organizing the data analyzed in this report.

\section{Author Contributions}

ST, PK, and PW designed the study. PK and SK acquired the data. ST analyzed and interpreted the data. ST were major contributors in writing the manuscript. All authors read and approved the final manuscript.

\section{References}

1. Lee JJ, Nguyen ET, Harrison JR, et al. Fluoroscopically guided caudal epidural steroid injections for axial low back pain associated with central disc protrusions: a prospective outcome study. Int Orthop 2019;43:1883-9.

2. Nandi J, Chowdhery A. A randomized controlled clinical trial to determine the effectiveness of caudal epidural steroid injection in lumbosacral sciatica. J Clin Diagn Res 2017;11:RC04-8.

3. Hirabayashi Y, Shimizu R, Matsuda I, Inoue S. Effect of extradural compliance and resistance on spread of extradural analgesia. Br J Anaesth 1990;65:508-13.

4. Singh V, Manchikanti L. Role of caudal epidural injections in the management of chronic low back pain. Pain Physician 2002;5:133-48.

5. Lee GY, Lee JW, Choi HS, Oh KJ, Kang HS. A new grading system of lumbar central canal stenosis on MRI: an easy and reliable method. Skeletal Radiol 2011;40:1033-9.

6. Azimi P, Mohammadi HR, Benzel EC, Shahzadi S, Azhari S. Lumbar spinal canal stenosis classification criteria: a new tool. Asian Spine J 2015;9:399-406.

7. Huskisson EC. Measurement of pain. Lancet 1974;2:1127-31.

8. Botwin KP, Gruber RD, Bouchlas CG, et al. Fluoroscopically guided lumbar transformational epidural steroid injections in degenerative lumbar stenosis: an outcome study. Am J Phys Med Rehabil 2002;81:898905.

9. Kim YC, Lim YJ, Lee SC. Spreading pattern of epidurally administered contrast medium in rabbits. Acta Anaesthesiol Scand 1998;42:1092-5.

10. Daly P. Caudal epidural analgesia in lumbosciatic pain. Anaesthesia 1970;25:346-8.

11. Kim KM, Kim HS, Choi KH, Ahn WS. Cephalic spreading levels after volumetric caudal epidural injections in chronic low back pain. J Korean Med Sci 2001;16:193-7. 
12. Murphy DT, Kavanagh EC, Poynton A, Chan VO, Moynagh MR, Eustace S. MR epidurography: distribution of injectate at caudal epidural injection. Skeletal Radiol 2015;44:565-71.

13. Nishimura N, Kitahara T, Kusakabe T. The spread of lidocaine and I-131 solution in the epidural space. Anesthesiology 1959;20:785-8.

14. Kushner FH, Olson JC. Retinal hemorrhage as a con- sequence of epidural steroid injection. Arch Ophthalmol 1995;113:309-13.

15. Purdy EP, Ajimal GS. Vision loss after lumbar epidural steroid injection. Anesth Analg 1998;86:119-22.

16. Karppinen J, Malmivaara A, Kurunlahti M, et al. Periradicular infiltration for sciatica: a randomized controlled trial. Spine (Phila Pa 1976) 2001;26:105967. 